\title{
Observation of Transient and Asymptotic Driven Structural States of Tungsten Exposed to Radiation
}

\author{
Daniel R. Mason $\odot,{ }^{1, *}$ Suchandrima Das $\odot,{ }^{2, \dagger}$ Peter M. Derlet, ${ }^{3, *}$ Sergei L. Dudarev ${ }^{1, \S}$ Andrew J. London ${ }^{1,}{ }^{1}$ \\ Hongbing Yu, ${ }^{2}$ Nicholas W. Phillips $\odot,{ }^{2}$ David Yang $\odot,{ }^{2}$ Kenichiro Mizohata, ${ }^{4}$ Ruqing $X u,{ }^{5}$ and Felix Hofmann $\odot^{2, \|}$ \\ ${ }^{1}$ UK Atomic Energy Authority, Culham Science Centre, Oxfordshire OX14 3DB, United Kingdom \\ ${ }^{2}$ Department of Engineering Science, University of Oxford, Parks Road, OX1 3PJ, United Kingdom \\ ${ }^{3}$ Condensed Matter Theory Group, Paul Scherrer Institut, CH-5232 Villigen PSI, Switzerland \\ ${ }^{4}$ University of Helsinki, P.O. Box 64, 00560 Helsinki, Finland \\ ${ }^{5}$ Advanced Photon Source, Argonne National Lab, 9700 South Cass Avenue, Argonne, Illinois 60439, USA
}

(Received 23 July 2020; revised 24 September 2020; accepted 2 October 2020; published 24 November 2020)

\begin{abstract}
Combining spatially resolved x-ray Laue diffraction with atomic-scale simulations, we observe how ionirradiated tungsten undergoes a series of nonlinear structural transformations with increasing radiation exposure. Nanoscale defect-induced deformations accumulating above 0.02 displacements per atom (dpa) lead to highly fluctuating strains at $\sim 0.1 \mathrm{dpa}$, collapsing into a driven quasisteady structural state above $\sim 1 \mathrm{dpa}$. The driven asymptotic state is characterized by finely dispersed vacancy defects coexisting with an extended dislocation network and exhibits positive volumetric swelling, due to the creation of new crystallographic planes through self-interstitial coalescence, but negative lattice strain.
\end{abstract}

DOI: 10.1103/PhysRevLett.125.225503

The effects of irradiation on materials and their implications for structural integrity are major concerns for the design and operation of advanced nuclear power reactors [1,2]. Direct mechanistic models can correlate the evolution of irradiation-induced residual stresses and strains with components' lifetime [3,4]; however, the dynamics of the damage microstructure are complex and nonlinear, span multiple length and timescales, and vary with exposure and environmental conditions $[5,6]$. It remains challenging to account for contributing factors at relevant length and timescales with a minimum-parameter model.

Quantitative experimental observations of irradiation effects require samples formed under controlled conditions of exposure, temperature, and applied stress. Ion irradiation offers a cost- and time-effective alternative to neutron irradiation avoiding sample activation [7], and real-space observations of microstructure produced by ion irradiation have contributed extensively to the development of highly irradiation-resistant materials $[1,8,9]$. Experimental techniques sensitive to the few-micron-thick ion damaged layer include transmission electron microscopy (TEM) [10-17], $\mathrm{x}$-ray diffraction [18-20], positron annihilation spectroscopy [21,22], micromechanical tests [5,23-25], and laserbased techniques [26-30].

Published by the American Physical Society under the terms of the Creative Commons Attribution 4.0 International license. Further distribution of this work must maintain attribution to the author(s) and the published article's title, journal citation, and DOI.
Transferable interpretation of ion-irradiated materials data is an outstanding challenge. Quantitative models for irradiation effects are restricted to pure crystalline materials and very low exposure, $10^{-6}$ to $10^{-4}$ displacements per atom (dpa) [31,32]. At high doses, consistent and unambiguous analysis proves difficult, and the interpretation of experiments relies on temperature-dose rate scaling [7], rate theory [33], or cluster dynamics [34,35]. These models use kinetic equations involving potentially a multitude of parameters and do not treat the microscopic fluctuating stresses and strains that drive defect interactions at the nanoscale [36-38].

The spatial variation of strains and stresses observed in irradiated materials $[39,40]$ can directly validate real-space simulations, since elasticity equations relate atomic-scale defects to macroscopic strains [4]. Here, we demonstrate this principle using an effectively parameter-free model to capture the physics of defect microstructure evolution without an overreliance on thermal activation. The 3D depth-resolved lattice strain induced by the entire population of irradiation defects is probed with $\sim 10^{-4}$ strain sensitivity using synchrotron x-ray microbeam Laue diffraction and interpreted quantitatively by direct atomic level simulations. The approach offers a unique advantage over TEM observations that only image defects larger than a critical size $[10,24,41,42]$.

Tungsten, the front-runner candidate for armor components in the International Thermonuclear Experimental Reactor $[43,44]$, serves as the prototype material for this study. In service, tungsten is anticipated to encounter significant radiation exposure [45]. The dose-dependent 
irradiation-induced defect microstructure in tungsten, under realistic operating conditions, is key to determining component lifetime and power plant availability. Currently, detailed qualitative information about microstructure is fragmented, particularly at ambient temperature for dense defect populations [5] where the mobility of defects is suppressed, resulting in exceedingly long relaxation times $[46,47]$. Here, we show how the nonlinear evolution of microstructure can be understood quantitatively by a systematic experimental and simulation study of ionirradiated tungsten exposed to a wide range of doses at room temperature.

Experimental observations.-Tungsten samples were irradiated with self-ions to damage levels from 0.001 to 10 dpa. The details of the sample preparation, ionimplantation method, and fluences used are provided in the Supplemental Material [48], with references to Refs. [38,49-51]. Target displacements and ion ranges estimated using the SRIM code [50,51] show a $\sim 2.5 \mu \mathrm{m}$ thick implanted layer [Fig. 1(a)].

Three $\langle 001\rangle$ grains $(\sim 300 \mu \mathrm{m}$ size $)$ were identified in each implanted sample using electron backscattering diffraction. In each grain, the strain in the $\langle 001\rangle$ direction was measured using depth-resolved Laue diffraction with $\sim 10^{-4}$ strain sensitivity $[18,20,52]$. A polychromatic x-ray beam (7-30 keV) was focused to $2300 \mathrm{~nm}$ FWHM using Kirkpatrick-Baez mirrors, and the sample placed at the beam focus in $45^{\circ}$ reflection geometry. Diffraction patterns were recorded on an area detector $\sim 500 \mathrm{~mm}$ above the sample. A resolution of $\sim 500 \mathrm{~nm}$ along the incident beam direction was achieved using the differential aperture x-ray microscopy technique [18,53-55].

A 3D reciprocal space map of each $(00 n)$ reflection was measured by monochromating the incident beam $\left(\Delta E / E \sim 10^{-4}\right)$ and scanning the photon energy $[18,56]$. More information about the diffraction measurements is provided in the Supplemental Material [48]. Figure 1(b) shows the diffracted intensity, integrated over the tangential reciprocal space directions, plotted as a function of the scattering vector magnitude $|\boldsymbol{q}|$ and depth in the sample. The broad peak between 0 and $\sim 2.5 \mu \mathrm{m}$ corresponds to the implanted layer, whereas the sharp peak at $\gg 2.5 \mu \mathrm{m}$ corresponds to undamaged material. The measured implanted layer thickness is in good agreement with the SRIM prediction.

Using the Laue data, we determine the lattice strain component normal to the sample surface. The peak center $q_{\text {fit }}(d)$ is found as a function of the depth using the center-ofmass method. In the small strain approximation, the lattice strain is then $\epsilon_{z z}(d)=q_{0} / q_{\mathrm{fit}}(d)-1$, where $q_{0}$ is the peak position for the reflection in an unstrained crystal found here for each measurement using the average peak position in the last $1.5 \mu \mathrm{m}$ depth [e.g., $d>11 \mu \mathrm{m}$ in Fig. 1(b)].

To plot strain as a function of the dose, we average the depth-dependent strain over the $2.5 \mu \mathrm{m}$ implanted layer
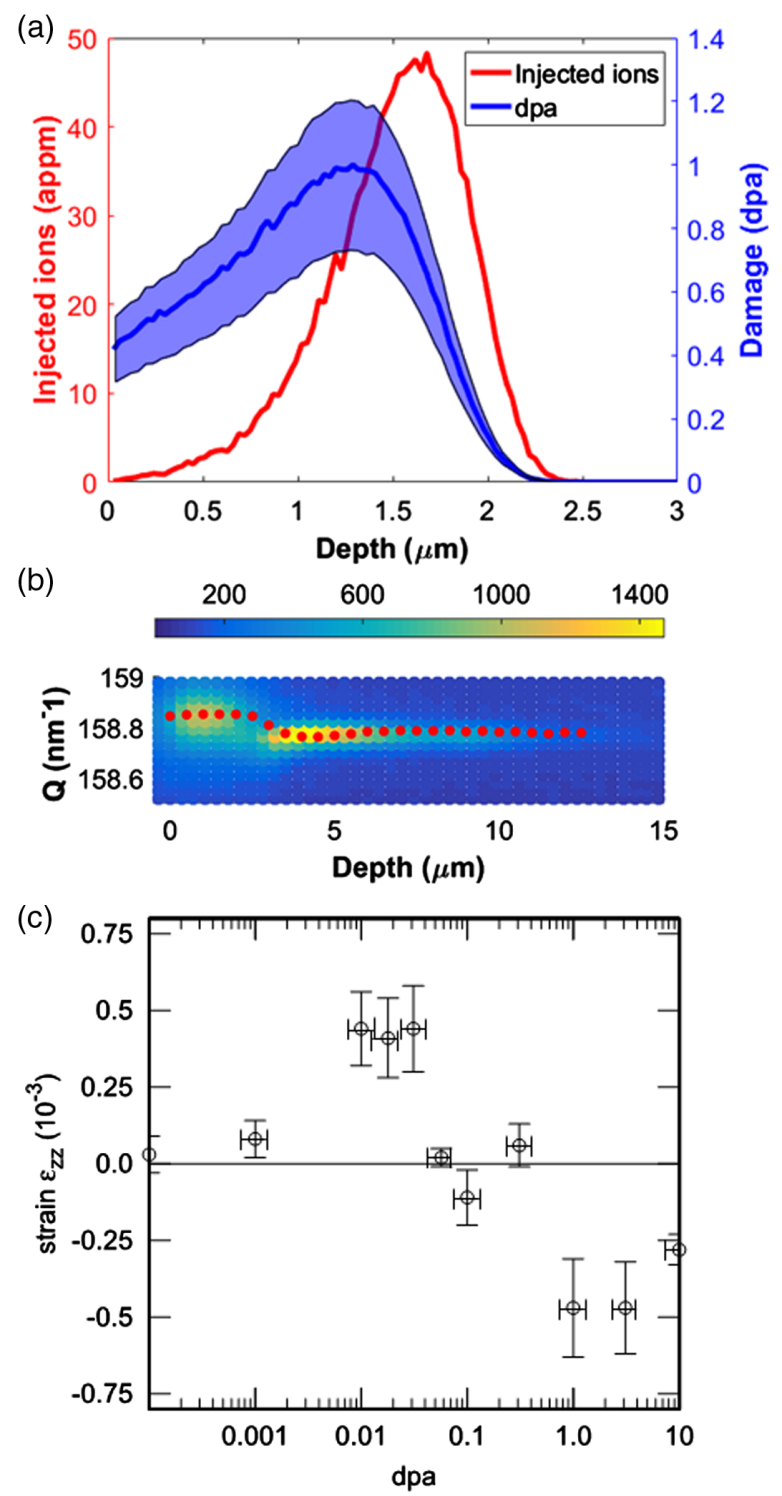

FIG. 1. (a) Injected tungsten ion concentration and displacement damage calculated using SRIM for the 1 dpa sample. The blue solid line shows the nominal dpa predicted using a threshold displacement energy of $68 \mathrm{eV}$. The shaded region shows upper and lower dpa bounds corresponding to threshold displacement energies of 55 and $90 \mathrm{eV}$, respectively. (b) Diffracted x-ray intensity integrated in the tangential reciprocal space directions for the (008) Bragg peak of the 1 dpa tungsten sample. Intensity is shown as a function of the scattering vector magnitude $|\mathbf{q}|$ and sample depth. The superimposed red dotted line shows the fitted peak centers $q_{\text {fit }}(d)$. (c) Depth-averaged strain measured in ion implantation experiments. Horizontal error bars indicate the dpa uncertainty associated with the variation of assumed threshold displacement energies.

[Fig. 1(c)]. Strain in the 0.001 dpa sample is very small. At low fluence, between 0.01 and $0.032 \mathrm{dpa}$, lattice expansion is observed. A transition occurs between 0.056 and $0.32 \mathrm{dpa}$, where the implantation-induced strains nearly vanish. At higher fluence ( $>1 \mathrm{dpa})$, we observe an 
apparent lattice contraction manifested as negative lattice strain. This suggests a highly unusual dose-dependent change in the defect microstructure over the exposure interval spanned by the observations. We note that the dpa uncertainty associated with the choice of threshold displacement energy in SRIM calculations is small compared to the explored damage range [Fig. 1(c)].

Simulations and interpretations.-To interpret experimental observations at the fundamental level of defect microstructure, we performed Frenkel pair creation and relaxation simulations $[21,57,58]$ using the creation relaxation algorithm (CRA) of Ref. [58]. Each step of the algorithm randomly selects a number of atoms and randomly displaces them to new positions within the simulation cell. The structure is relaxed using LAMMPS [59] with an empirical potential for tungsten [60], under three dimensional periodic boundary conditions with zero stress condition in the $\hat{z}$ direction (oriented with [001]) and zero strain in the $x-y$ plane, reflecting the bulk constraint imposed by the substrate.

This process is repeated many times and results in a microstructure that begins with isolated vacancy and interstitial defects and evolves via interstitial dislocation loop nucleation and coalescence to an extended dislocation network. The ratio of Frenkel pairs inserted into total atom content is the canonical dpa dose (cdpa) [58]. Representative results in Fig. 2 show realizations of the microstructure at 0.05 and 0.3 cdpa. At $0.05 \mathrm{cdpa}$, the developing internal stress field has driven some of the interstitials to nucleate into
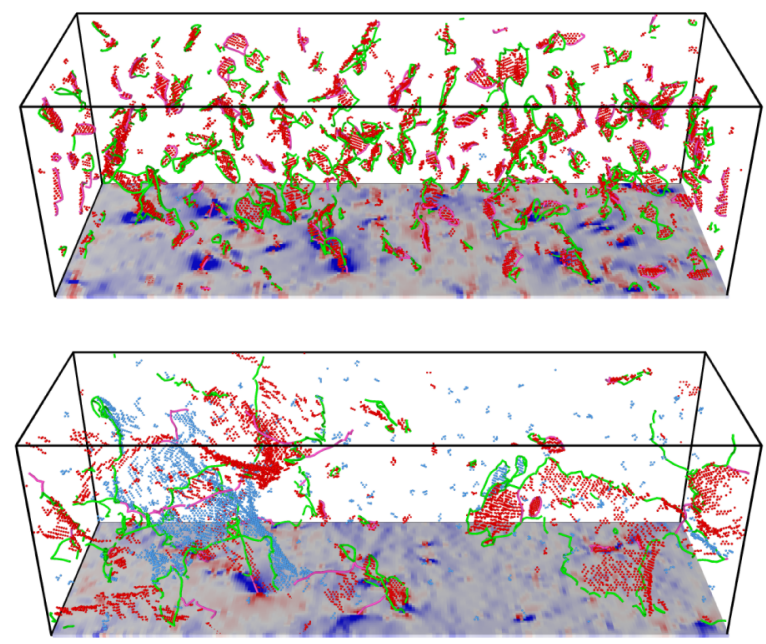

FIG. 2. Representative Frenkel pair insertion simulations at 0.05 (top) and 0.3 (bottom) dpa using the CRA algorithm. The box size is $20.2 \times 20.2 \times 63.2 \mathrm{~nm}^{3}$, and the unconstrained cell dimension $\hat{z}$ is horizontal. Vacancy (blue) and interstitial (red) clusters with $>3$ point defects are shown. Note the apparent formation of vacancy loops. A superimposed dislocation extraction algorithm analysis [62] shows both $1 / 2\langle 111\rangle$ (green) and $\langle 100\rangle$ (pink) dislocation lines. In the $y=0$ plane, the strain tensor component $\epsilon_{z z}$ is shown, with color scale blue, white, red representing $-5 \%, 0,+5 \%$ strain, respectively. dislocation loops, which by 0.3 cdpa have coalesced to extended dislocation structures, resulting in a microstructure that is insensitive to further Frenkel pair insertion [58]. Additional information about the atomistic simulations can be found in the Supplemental Material [48], with references to Refs. [58,60,61].

Frenkel pair insertion is a drastic simplification of the $20 \mathrm{MeV}$ self-ion cascades used in experiment $[63,64]$ but predicts microstructures qualitatively similar to overlapping molecular dynamics cascade simulations $[58,65]$. It should be noted that there is no thermal activation in CRA simulations-all relaxation is stress driven-so CRA describes microstructures where long-range diffusion does not occur. For the present case of high-purity, lowtemperature tungsten, vacancy migration is inactive [66]. The strong asymmetry in athermal mobility between vacancies and interstitials is therefore a justifiable physical limit and central to the observed simulated structural evolution. However, for materials that contain defect structures (impurities, sessile dislocation structures, etc.) that hinder interstitial mobility $[38,67]$ and reduce this asymmetry, the situation is less clear but addressable using a combination of dedicated experiments and CRA simulations as done here.

As in experiment, a measure of lattice strain can be obtained from a diffraction pattern, which for the case of simulation can be determined straightforwardly from the atomic positions of the microstructure produced by the Frenkel insertion method. Kinematic diffraction theory gives the diffraction spot intensity as being proportional to the square of the structure factor $I(q) \propto|S(q)|^{2}$, where

$$
S(q)=1 / \sqrt{N} \sum_{j} \exp \left[i q z_{j}\right]
$$

Here, both $q$ and $z_{j}$ are along the out-of-plane $z$ direction with the latter being the $z$ position of atom $j$. We use the simulated [002] spot to find $q_{\text {fit }}$ and hence the lattice strain as above. The resulting lattice strain is plotted in Fig. 3(a) as a function of the cdpa and demonstrates similar behavior to that seen in experiment, peaking at a cdpa of 0.05 after which it becomes negative at higher values of cdpa. While there is remarkably good quantitative agreement as a function of the dose, the scale of the simulated lattice strain is an order of magnitude larger than in experiments. This difference may be attributed to the absence of structural relaxation arising from thermal fluctuations [3].

Figure 3(a) also plots the volumetric strain associated with the change in volume of the simulation cell defined as $\epsilon_{\mathrm{vol}}=L / L_{0}-1$. Here, $L$ is the evolving simulation cell periodic length along the $z$ direction. The volumetric strain initially follows the lattice strain, indicating that it arises directly from a homogeneous lattice expansion, which in this case is due to the low dose microstructural regime of lattice interstitials and vacancies. However, at doses of 


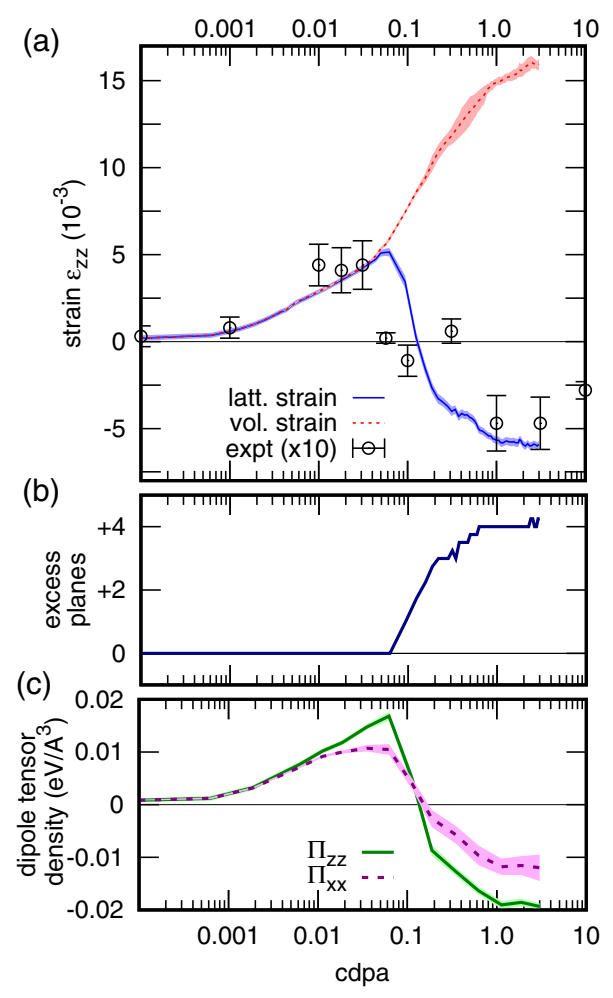

FIG. 3. (a) Lattice strain and volumetric strain (dashed) derived from simulations. Shaded region denotes 1 standard deviation. The experimental strain data are scaled by a factor of 10 to compare trends as well as absolute values. Volumetric strain due to the injected self-ions is small. (b) Number of excess planes recorded in the simulation. (c) Defect dipole tensor density (see text) computed from simulation cell stress. Note the horizontal scale is the same for all three plots.

approximately $0.05 \mathrm{cdpa}$, the volumetric strain decouples from the lattice strain and continues to increase with the dose. In this regime, interstitials cluster to form dislocation loops that grow in size and eventually coalesce, resulting in the creation of new crystal planes along the $z$ direction seen in Fig. 3(b). This process preserves the increase in volume due to interstitial defects, while converting metastable microstructure into near-perfect crystal. This observation agrees with reports in other materials of lattice plane creation as a volumetric swelling mechanism [68,69]. The good agreement between these simulations and experiment allows us to conclude that the change in the sign of lattice strain observed in experiment should not be interpreted as a transition from irradiation-induced swelling to irradiation-induced contraction.

Such a decoupling between volumetric and lattice strains has been used to infer vacancy concentrations in metals [70] and has also been observed in simulations under bulk isotropic conditions [58]. The latter lead to a zero lattice strain at high doses, whereas in the present case symmetry breaking leads to an asymptotic energy minimum with net negative out-of-plane strain. The general high dose strain condition as a function of the sample boundary conditions, elastic constants, and defect densities needs further analysis.

Using the elastic dipole tensor formalism to represent defects as sources of stress [71], and taking into account the zero $x, y$-strain condition imposed by the substrate and the traction-free condition at the surface, we find the nonvanishing components of lattice strain and stress in the irradiated layer $\epsilon_{z z}=\left(\Pi_{z z} / 2 \mu\right)(1-2 \nu) /(1-\nu)$ and $\sigma_{x x}=\sigma_{y y}=\Pi_{x x}-\nu \Pi_{z z} /(1-\nu)$. Here, $\Pi_{i j}$ is the volume density of dipole tensors of defects $\Pi_{i j}(\mathbf{r})=$ $\sum_{a} p_{i j}^{(a)} \delta\left(\mathbf{r}-\mathbf{R}_{a}\right)$, and $\mu$ and $\nu$ are the shear modulus and the Poisson ratio of tungsten.

Computing $\epsilon_{z z}$ and $\sigma_{x x}$ from simulations, we find that the lattice strain sign change coincides with the observation in the simulated diffraction pattern of the start of the formation of additional atomic planes parallel to the surface; see Fig. 3(b). These planes, formed by the coalescence of interstitial dislocation loops, preserve the volumetric strain in the material, but, by converting the interstitial defect content into crystal planes, they reduce the lattice strain of the irradiated layer. This is confirmed by all components of the dipole density tensor becoming negative in the high dose limit.

The simulated microstructure beyond $1 \mathrm{dpa}$ is dominated by network dislocations, a small number of dislocation loops of both interstitial and vacancy type, and a large number of excess vacancies. The vacancy population totaling $2.5 \pm$ $0.1 \%$ lattice sites unoccupied leads to the observed net negative lattice strain. The smaller magnitude lattice strain seen experimentally is likely due to thermally activated defect recombination, an aspect not captured by the present atomistic simulations.

The anisotropy of the dipole tensor density emerging as a function of the dose is the result of the self-action of the uniaxial stress field developing in the irradiated layer on the population of defects at a dose above $\sim 0.1 \mathrm{dpa}$. Figures 4 (a)4(c) show how an isolated interstitial $\mathbf{b}=1 / 2\langle 111\rangle$ dislocation loop changes its habit plane in response to an applied uniaxial strain. The response stems from the minimization of the energy of interaction of each individual defect with strain $E=-p_{z z} \epsilon_{z z}$, where $p_{z z}$ is the $z z$ component of the dipole tensor of a defect, for example, a dislocation loop [72,73]. The average orientation of the habit plane $\hat{\mathbf{n}}$ of the interstitial loops and extended dislocation structures in our simulations is measured and plotted via $\langle\hat{\mathbf{n}} \cdot \hat{\mathbf{z}}\rangle$ as a function of the cdpa in the right panel of Fig. 4. This is done through numerically determining the optimal habit plane orientation of the dislocation structures identified by the planes of interstitials. The figure reveals that at low dose this favors the orientation of the habit planes of interstitial loops whose normals point in the out-of-plane direction, favoring the coalescence of loops into new atomic planes. On the other hand, in the high dose limit, where $\epsilon_{z z}<0$, the habit plane normal vectors of interstitial loops reorient, tending now to point more toward the in-plane direction. As a result, no additional atomic 

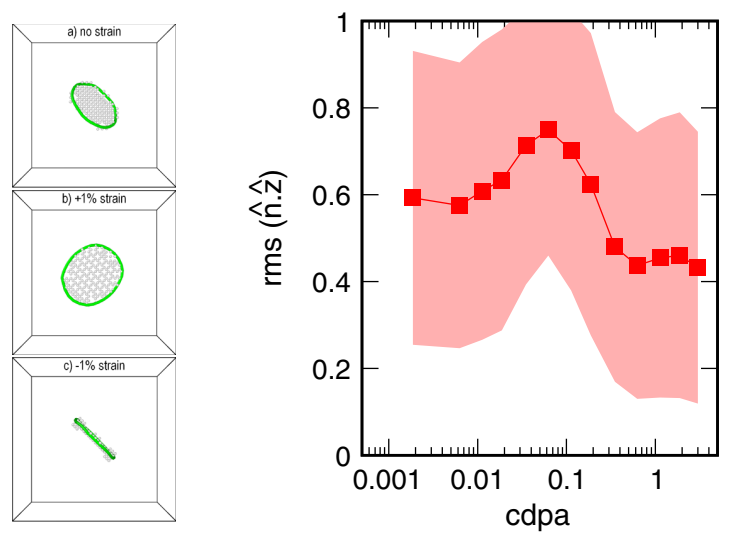

FIG. 4. Left: a 199-interstitial (4 nm diameter) glissile loop with $\mathbf{b}=1 / 2\langle 111\rangle$ relaxed under a small uniaxial strain (viewed along the strain axis) exhibits a spontaneous rotation of the habit plane with no change in $\mathbf{b}$. (a) corresponds to zero applied strain, whilst (b) and (c) correspond to applied strains of $+1 \%$ and $-1 \%$, respectively. The effect is more pronounced for larger loops as the dipole tensor of a loop is proportional to the loop vector area $[72,73]$. In projection onto the Burgers vector direction, the change of orientation of the loop is undetectable. This stems from the conservation of the relaxation volume of the loop (b - A ) [73], where $\mathbf{A}$ is the vector area of the loop. Right: root-mean-square orientation of habit plane normal as a function of the dose. Shaded region is 1 standard deviation of the population. Standard error is order symbol size.

crystal planes are formed beyond $\sim 0.6 \mathrm{dpa}$. It is noted that such a habit plane reorientation is a low barrier-energy process that occurs even under zero-loading conditions due to thermal fluctuations $[74,75]$.

The negative lattice strain developing in the high dose limit is therefore a nonlinear self-consistent phenomenon resulting from the interaction of radiation defects with the anisotropic uniaxial stress state developing in the irradiated layer.

Conclusions. - We find that upon ion irradiation of a tungsten surface layer, the measured out-of-plane lattice strain transitions from a positive to negative out-of-plane lattice strain. Through the use of the creation relaxation algorithm atomistic simulation method, this behavior is found to stem from the nonlinear self-consistent interaction of the radiation defect microstructure with its own stress field due to the constraint imposed by the unimplanted substrate material. The macroscopic volumetric strain, on the other hand, increases monotonically reflecting the wellknown phenomenon of irradiation-induced swelling. The observed effect is likely to be a fundamental common feature of ion-irradiation experiments offering a simple and direct way of assessing the effect of stress and strain fields on defects produced in materials by irradiation. The present results also highlight that high dose irradiation can induce significant internal elastic loading, leading to dimensional changes and radiation-induced creep, all of which can adversely affect material components during operation of advanced fission and fusion reactors.

Data from experiments and simulations presented in this Letter are available online at https://doi.org/10.5287/ bodleian:KZrJx17Zw.

This work was funded by Leverhulme Trust Research Project Grant No. RPG-2016-190. X-ray diffraction experiments used resources of the Advanced Photon Source, a U.S. Department of Energy (DOE) Office of Science User Facility operated for the DOE Office of Science by Argonne National Laboratory under Contract No. DEAC02-06CH11357. This work was carried out in part within the framework of the EUROfusion Consortium and has received funding from the Euratom research and training program 2019-2020 under Grant Agreement No. 633053 and from the Research Councils UK Energy Programme (Grant No. EP/T012250/1). We also acknowledge funding from the European Research Council under the European Union's Horizon 2020 research and innovation program (Grant Agreement No. 714697). The views and opinions expressed herein do not necessarily reflect those of the European Commission. This work was also partly supported by the École Polytechnique Fédérale de Lausanne Swiss Plasma Center. Ion implantations were performed at the Helsinki Accelerator Laboratory, Department of Physics, University of Helsinki. We would like to thank D. Perez, M. Boleininger, M. C. Marinica, M. J. Caturla, and P.-W. Ma for stimulating discussions.

\footnotetext{
*Daniel.Mason@ukaea.uk

†Suchandrima.Das@eng.ox.ac.uk

†Peter.Derlet@psi.ch

${ }^{\S}$ Sergei.Dudarev@ukaea.uk

felix.hofmann@eng.ox.ac.uk
}

[1] S. J. Zinkle and G. S. Was, Acta Mater. 61, 735 (2013).

[2] J. Knaster, A. Moeslang, and T. Muroga, Nat. Phys. 12, 424 (2016).

[3] J. Marian, C. S. Becquart, C. Domain, S. L. Dudarev, M. R. Gilbert, R. J. Kurtz, D. R. Mason, K. Nordlund, A. E. Sand, L. L. Snead et al., Nucl. Fusion 57, 092008 (2017).

[4] S. L. Dudarev, D. R. Mason, E. Tarleton, P.-W. Ma, and A. E. Sand, Nucl. Fusion 58, 126002 (2018).

[5] D. Kiener, P. Hosemann, S. A. Maloy, and A. M. Minor, Nat. Mater. 10, 608 (2011).

[6] G. S. Was, Fundamentals of Radiation Materials Science, Metals and Alloys, 2nd ed. (Springer-Verlag, New York, 2017).

[7] G. S. Was and R. S. Averback, in Comprehensive Nuclear Materials, edited by R. J. M. Konings (Elsevier, Amsterdam, 2012), Vol. 1, pp. 195-221.

[8] J. Silcox and P. B. Hirsch, Philos. Mag. 4, 72 (1959).

[9] R. E. Smallman and A. H. W. Ngan, in Modern Physical Metallurgy, 8th ed., edited by R. E. Smallman and A. H. W. 
Ngan (Butterworth-Heinemann, Oxford, 2014), pp. 251285.

[10] X. Yi, M. L. Jenkins, K. Hattar, P. D. Edmondson, and S. G. Roberts, Acta Mater. 92, 163 (2015).

[11] X. Yi, M. L. Jenkins, M. A. Kirk, Z. Zhou, and S. G. Roberts, Acta Mater. 112, 105 (2016).

[12] X. Yi, A. E. Sand, D. R. Mason, M. A. Kirk, S. G. Roberts, K. Nordlund, and S. L. Dudarev, Europhys. Lett. 110, 36001 (2015).

[13] O. El-Atwani, E. Esquivel, M. Efe, E. Aydogan, Y. Wang, E. Martinez, and S. Maloy, Acta Mater. 149, 206 (2018).

[14] L. Ciupiński, O. V. Ogorodnikova, T. Płociński, M. Andrzejczuk, M. Rasiński, M. Mayer, and K. J. Kurzydłowski, Nucl. Instrum. Methods Phys. Res., Sect. B 317, 159 (2013).

[15] W. Guo, S. Wang, L. Ge, E. Fu, Y. Yuan, L. Cheng, and G. H. Lu, Nucl. Fusion 60, 034002 (2020).

[16] R. W. Harrison, J. A. Hinks, and S. E. Donnelly, Scr. Mater. 150, 61 (2018).

[17] I. Ipatova, R. W. Harrison, S. E. Donnelly, M. J. Rushton, S. C. Middleburgh, and E. Jimenez-Melero, J. Nucl. Mater. 526, 151730 (2019).

[18] S. Das, W. Liu, R. Xu, and F. Hofmann, Mater. Des. 160, 1226 (2018).

[19] I. de Broglie, C. E. Beck, W. Liu, and F. Hofmann, Scr. Mater. 107, 96 (2015).

[20] F. Hofmann, D. Nguyen-Manh, M. R. Gilbert, C. E. Beck, J. K. Eliason, A. A. Maznev, W. Liu, D. E. J. Armstrong, K. A. Nelson, and S. L. Dudarev, Acta Mater. 89, 352 (2015).

[21] A. Debelle, M. Barthe, and T. Sauvage, J. Nucl. Mater. 376, 216 (2008).

[22] X. Hu, T. Koyanagi, M. Fukuda, Y. Katoh, L. L. Snead, and B. D. Wirth, J. Nucl. Mater. 470, 278 (2016).

[23] D. E. J. Armstrong, X. Yi, E. A. Marquis, and S. G. Roberts, J. Nucl. Mater. 432, 428 (2013).

[24] T. Hwang, M. Fukuda, S. Nogami, A. Hasegawa, H. Usami, K. Yabuuchi, K. Ozawa, and H. Tanigawa, Nucl. Mater. Energy 9, 430 (2016).

[25] P. Hosemann, D. Kiener, Y. Wang, and S. A. Maloy, J. Nucl. Mater. 425, 136 (2012).

[26] M. P. Short, C. A. Dennett, S. E. Ferry, Y. Yang, V. K. Mishra, J. K. Eliason, A. Vega-Flick, A. A. Maznev, and K. A. Nelson, JOM 67, 1840 (2015).

[27] F. Hofmann, D. R. Mason, J. K. Eliason, A. A. Maznev, K. A. Nelson, and S. L. Dudarev, Sci. Rep. 5, 16042 (2015).

[28] C. A. Dennett, D. L. Buller, K. Hattar, and M. P. Short, Nucl. Instrum. Methods Phys. Res., Sect. B 440, 126 (2019).

[29] C. A. Dennett, K. P. So, A. Kushima, D. L. Buller, K. Hattar, and M. P. Short, Acta Mater. 145, 496 (2018).

[30] A. Reza, H. Yu, K. Mizohata, and F. Hofmann, Acta Mater. 193, 270 (2020).

[31] C. C. Fu, J. Dalla Torre, F. Willaime, J.-L. Bocquet, and A. Barbu, Nat. Mater. 4, 68 (2005).

[32] C. J. Ortiz and M. J. Caturla, J. Comput.-Aided Mater. Des. 14, 171 (2007).

[33] T. Jourdan and J.-P. Crocombette, Phys. Rev. B 86, 054113 (2012).

[34] J. Marian and V. V. Bulatov, J. Nucl. Mater. 415, 84 (2011).

[35] A. Y. Dunn, L. Capolungo, E. Martinez, and M. Cherkaoui, J. Nucl. Mater. 443, 128 (2013).
[36] P. Anderson, J. Hirth, and J. Lothe, Theory of Dislocations, 3rd ed. (Cambridge University Press, Cambridge, England, 2017).

[37] S. L. Dudarev, M. R. Gilbert, K. Arakawa, H. Mori, Z. Yao, M. L. Jenkins, and P. M. Derlet, Phys. Rev. B 81, 224107 (2010).

[38] D. R. Mason, X. Yi, M. A. Kirk, and S. L. Dudarev, J. Phys. Condens. Matter 26, 375701 (2014).

[39] N. Phillips, H. Yu, S. Das, D. Yang, K. Mizohata, W. Liu, R. Xu, R. Harder, and F. Hofmann, Acta Mater. 195, 219 (2020).

[40] H. Yu, P. Karamched, S. Das, J. Liu, K. Mizohata, and F. Hofmann, arXiv:2005.09788.

[41] Z. Zhou, M. L. Jenkins, S. L. Dudarev, A. P. Sutton, and M. A. Kirk, Philos. Mag. 86, 4851 (2006).

[42] X. Yi, M. L. Jenkins, M. A. Kirk, Z. Zhou, and S. G. Roberts, Acta Mater. 112, 105 (2016).

[43] M. Rieth, S. L. Dudarev, S. M. Gonzalez De Vicente, J. Aktaa, T. Ahlgren, S. Antusch, D. E. J. Armstrong, M. Balden, N. Baluc, M.-F. Barthe et al., J. Nucl. Mater. 432, 482 (2013).

[44] J. Reiser and A. Hartmaier, Sci. Rep. 10, 2739 (2020).

[45] M. R. Gilbert, S. L. Dudarev, S. Zheng, L. W. Packer, and J.-C. Sublet, Nucl. Fusion 52, 083019 (2012).

[46] F. Ferroni, X. Yi, K. Arakawa, S. P. Fitzgerald, P. D. Edmondson, and S. G. Roberts, Acta Mater. 90, 380 (2015).

[47] K. Papamihail, K. Mergia, F. Ott, Y. Serruys, T. Speliotis, G. Apostolopoulos, and S. Messoloras, Phys. Rev. B 93, 100404(R) (2016).

[48] See Supplemental Material at http://link.aps.org/ supplemental/10.1103/PhysRevLett.125.225503 for details of sample preparation, experimental measurements, and atomistic simulations.

[49] P. Tikkanen, V. Palonen, H. Jungner, and J. Keinonen, Nucl. Instrum. Methods Phys. Res., Sect. B 223-224, 35 (2004).

[50] ASTM International Report No. ASTM E52196 e1, 2009, https://dx.doi.org/10.1520/E0521-96R09E01.

[51] J. F. Ziegler and J. P. Biersack, Nucl. Instrum. Methods Phys. Res., Sect. B 268, 1818 (2010).

[52] S. Das, D. E. J. Armstrong, Y. Zayachuk, W. Liu, R. Xu, and F. Hofmann, Scr. Mater. 146, 335 (2018).

[53] F. Hofmann, B. Abbey, W. Liu, R. Xu, B. F. Usher, E. Balaur, and Y. Liu, Nat. Commun. 4, 2774 (2013).

[54] W. Liu, P. Zschack, J. Tischler, G. Ice, and B. Larson, AIP Conf. Proc. 1365, 108 (2010).

[55] B. C. Larson, W. Yang, G. E. Ice, J. D. Budai, and J.Z. Tischler, Nature (London) 415, 887 (2002).

[56] K. S. Chung and G. E. Ice, J. Appl. Phys. 86, 5249 (1999).

[57] A. Chartier, C. Onofri, L. Van Brutzel, C. Sabathier, O. Dorosh, and J. Jagielski, Appl. Phys. Lett. 109, 181902 (2016).

[58] P. M. Derlet and S. L. Dudarev, Phys. Rev. Mater. 4, 023605 (2020).

[59] S. Plimpton, J. Comput. Phys. 117, 1 (1995).

[60] D. R. Mason, D. Nguyen-Manh, and C. S. Becquart, J. Phys. Condens. Matter 29, 505501 (2017).

[61] D. Mason, D. Nguyen-Manh, M.-C. Marinica, R. Alexander, A. Sand, and S. L. Dudarev, J. Appl. Phys. 126, 075112 (2019).

[62] A. Stukowski, V. V. Bulatov, and A. Arsenlis, Model. Simul. Mater. Sci. Eng. 20, 085007 (2012). 
[63] A. E. Sand, D. R. Mason, A. De Backer, X. Yi, S. L. Dudarev, and K. Nordlund, Mater. Res. Lett. 5, 357 (2017).

[64] A. Sand, J. Byggmästar, A. Zitting, and K. Nordlund, J. Nucl. Mater. 511, 64 (2018).

[65] F. Granberg, J. Byggmästar, and K. Nordlund, J. Nucl. Mater. 528, 151843 (2020).

[66] D. Nguyen-Manh, A. P. Horsfield, and S. L. Dudarev, Phys. Rev. B 73, 020101(R) (2006).

[67] A. Bakaev, A. Zinovev, D. Terentyev, G. Bonny, C. Yin, N. Castin, Y. A. Mastrikov, and E. E. Zhurkin, J. Appl. Phys. 126, 075110 (2019).

[68] A. D. Brailsford and R. Bullough, Phil. Trans. R. Soc. A 302, 87 (1981).
[69] C. H. Woo and B. N. Singh, Phys. Status Solidi (b) 159, 609 (1990).

[70] R. Simmons and R. Balluffi, Phys. Rev. 117, 52 (1960).

[71] G. Leibfried and N. Breuer, Point Defects in Metals (Springer, Berlin, 1978).

[72] W. G. Wolfer, T. Okita, and D. M. Barnett, Phys. Rev. Lett. 92, 085507 (2004).

[73] S. L. Dudarev and P.-W. Ma, Phys. Rev. Mater. 2, 033602 (2018).

[74] Y. N. Osetsky, A. Serra, B. N. Singh, and S. I. Golubov, Philos. Mag. A 80, 2131 (2000).

[75] P. M. Derlet, M. R. Gilbert, and S. L. Dudarev, Phys. Rev. B 84, 134109 (2011). 